\title{
Reducing bias: accounting for the order of co-first authors
}

\author{
Reflecting an increasing emphasis on collaborative science, the number of \\ authors on published articles has markedly risen with time. With this trend, \\ we see an increase in papers designating 2 or more co-first authors. To \\ improve transparency in how such designations are made and reduce bias in \\ the assignment of order, the $\mathrm{JCl}$ is now requiring an explanation for how the \\ first-author position is determined when shared among contributing authors.
}

Today, the overwhelming majority of biomedical science publications have more than 1 author, and the number of authors per publication is rising exponentially as a function of time (1). For multiauthored papers in the biomedical sciences, the order in which authors are listed is a code for conveying the importance of their contributions. In this scheme, the first position is typically assumed by the individual (usually a junior person in training) who does the bulk of the work in the study, whereas the last position is taken by a senior person who has supervised the work. Hence, the author position is critically important for attributing contributions from junior investigators who need first-author publications to further their careers in biomedical research. This mechanism for credit attribution worked reasonably well when there were 2 authors but has become increasingly strained as research groups have grown to produce complex studies with numerous authors making important contributions. Consequently, recent years have witnessed a practice whereby the first or last author positions in the byline are shared by 2 or more individuals, often with an asterisk indicating that "these individuals contributed equally" (2). Since only 1 author can be listed first on a publication, the chosen individual among a list of "equally contributing authors" is likely to garner a greater share of citation credit and recognition from readers by virtue of having their name first.

Two recent studies suggest that the author order is not equitable with regards to sex for authors claiming equal contributions $(2,3)$. If 2 individuals contribute equally and/or agree to share the first position in an article, then one could assume that, in a fair world, the likelihood of males and females being listed first in the author byline would be 50:50. Unfortunately, this does not appear to be the case. A study of sex and author positions in the pediatric literature found a significant deficit of females heading the author byline, even when they contributed equally (3). Another study of over 3000 biomedical publications found preferences for males occupying the first position in the author byline over the past 2 decades, although the frequency was approaching equal numbers in recent years (4). Both studies raise the specter of sex bias skewing how authors are listed in the first position among coauthors. In the first 3 issues of 2019 , a third of our research publications (28/84) listed 2 or more authors as co-first authors or had equally contributing authors in the first 2 author positions. Twelve of these publications listed 3 or more authors as co-first/equally contributing authors, and 1 paper contained an unusually high total of 9 co-first authors. JCI does not collect information about the sex of our authors, but by using publicly available information to estimate sex of individuals in the first 2 author positions, we found that order was malefemale in 11 cases, female-male in 4 cases, male-male in 6 cases, female-female in 4 cases, and undetermined in 3 cases. Hence, the experience at JCI in the early months of 2019 is consistent with those 2 reports.

The JCI is committed to the highest publication standards, and that includes doing what it can to reduce sex inequity or other biases. Consequently, we already ask that authors describe their contributions for studies published in our journal. Listing author contributions provides a mechanism for individuals to clarify how they contributed to the study, which can help when attributing credit in multiauthored publications. Going forward, we will require that senior/corresponding authors state the method used in assigning the first-author position among coauthors. Furthermore, we will discourage the use of the phrase "contributed equally," which is vague and likely untrue except in the very rare situation that 2 individuals truly made the same contribution to a study. Instead, the JCI will use the wording that 2 or more authors share a specific author position. By requiring information of how the author position was selected when individuals share a position on the author byline, we are hoping to provide more information that can be used in attributing credit. We are not naive to believe that these requirements will solve the problem of sex bias in choosing author order, but we are hopeful that they will trigger discussions between authors and their supervisors that could lead to fairer choices. When individuals make such critical contributions to a study such that they merit sharing an important author byline position, the first author could be determined alphabetically or by flipping a coin, accompanied by an explicit statement regarding the method used for author ordering. Alternatively, we are open to considering other mechanisms for selecting the author order. Ultimately, the journal does not determine authorship order, but we encourage all of our authors to consider the recommendations of the International Committee of Medical Journal Editors (5) for defining authorship, and our policies seek to promote transparency and accountability in determining author order. In order to foster fairness in "team science," we believe that review and promotion committees will be more likely to give equal credit when they understand which methods are used for author ordering and when they realize that this transparency has to be good for all authors and, by extension, for science as a whole.

Arturo Casadevall, Gregg L. Semenza, Sarah Jackson, Gordon Tomaselli, and Rexford S. Ahima 
1. Medline/Pubmed Resources. Number of authors per MEDLINE/PubMed citation. NIH website. https://www.nlm.nih.gov/bsd/authors1.html. Accessed on March 20, 2019.

2. Tscharntke T, Hochberg ME, Rand TA, Resh VH, Krauss J. Author sequence and credit for contributions in multiauthored publications. PLOS Biol. 2007;5(1):e18.

3. Fishman M, Williams WA, Goodman DM, Ross LF. Gender Differences in the authorship of original research in pediatric journals, 2001-2016. JPediatr. 2017;191:244-249.e1.

4. Broderick NA, Casadevall A. Gender inequalities among authors who contributed equally. Elife. 2019;8:e36399.

5. International Committee of Medical Journal Editors (ICJME). Defining the role of authors and contributors. ICJME website. http://www. icmje.org/recommendations/browse/roles-andresponsibilities/defining-the-role-of-authors-andcontributors.html. Accessed on March 20, 2019. 